\title{
HUMAN PLACENTAL LIPID PEROXIDATION. SOME CHARACTERISTICS OF THE NADPH-SUPPORTED MICROSOMAL REACTION
}

\author{
A. P. KULKarNi* and M. F. Kenel $\dagger$ \\ Toxicology Program, Department of Environmental and Industrial Health, School of Public Health, \\ The University of Michigan, Ann Arbor, MI 48109-2029, U.S.A.
}

(Received 11 November 1986)

\begin{abstract}
The evidence presented in this paper indicates the existence of NADPH-supported lipid peroxidation in human placental microsomes. Thiobarbituric acid assay was used to estimate quantitatively lipid peroxidation.

2. Several biochemical characteristics of the reaction were examined. Maximal lipid peroxidation occurred at pH 7.4 and at a protein concentration of approx. $0.2 \mathrm{mg}$ microsomal protein $/ \mathrm{ml}$. The presence of NADPH and chelated iron was required. The reaction was linear up to $5 \mathrm{~min}$ and did not exhibit an initial lag phase.

3. Under optimal assay conditions, the rate of lipid perioxidation ranged from 2 to $6 \mathrm{nmol}$ malondialdehyde formed $/ \mathrm{min} / \mathrm{mg}$ protein in different preparations of placental microsomes.

4. Inconclusive results were obtained when assays were performed in the presence of scavengers of reactive oxygen species.

5. Marked inhibition in the malondialdehyde accumulation was observed when phosphate buffer was added to the incubation media.

6. This inhibitory effect appeared to be due to the removal of chelated iron from the system and not due to interference with the electron transport mechanism.
\end{abstract}

\section{INTRODUCTION}

Previous reports have shown that human placentas of non-smoking women contain very little of cytochrome $P-450$ which is capable of xenobiotic metabolism (Juchau, 1980). In view of this, our laboratory is exploring alternate pathways of xenobiotic oxidation in this tissue. Earlier, we reported the activities of flavin containing monooxygenase (Osimitz and Kulkarni, 1982), indanol dehydrogenase (Kulkarni et al., 1985), and peroxidase (Nelson and Kulkarni, $1986 \mathrm{a}, \mathrm{b}$ ) in either human or mouse placentas.

Recent reports have documented that rat liver microsomes are capable of co-oxidation of xenobiotics during ascorbate- or NADPH-supported lipid peroxidation (Morgenstern et al., 1981; Dix and Marnett, 1983). In light of these studies, and the facts that the generation of reactive oxygen species due to cellular redox activities and concomitant low level of lipid peroxidation are the normal biochemical events in different mammalian tissues (Kulkarni and Hodgson, 1981), we are currently evaluating lipid peroxidation-coupled co-oxidation as a potential alternate pathway of xenobiotic metabolism in human placenta.

It is well established that various biomembranes are susceptible to lipid peroxidation which impairs the integrity of their structure and function (Plaa and Witschi, 1976; Slater, 1984; Halliwell and Gutteridge,

*To whom reprint requests should be addressed.

†Present address: McNeil Pharmaceutical, Spring House, PA 19477, U.S.A.
1984; Fridovich, 1986). Lipid peroxidation has been implicated as a major mechanism of toxicity of certain xenobiotics and drugs (Plaa and Witschi, 1976; Bus and Gibson, 1979; Slater, 1984; Aust et al., 1985). Exposure to environmental chemicals capable of stimulating lipid peroxidation within placental membranes in vivo may be of toxicological significance, since it may alter the placental biochemistry in such a way that the fetus would be at risk. Besides this indirect mode of reactive oxygen toxicity, the possibility exists for a direct exposure of fetus to the relatively stable cytotoxic agents generated during lipid peroxidation (Benedetti et al., 1984) in placenta after translocation.

The reports on the subject of placental lipid peroxidation are inconsistent. Although the occurrence of ascorbate supported non-enzymatic lipid peroxidation has been repeatedly demonstrated in human placenta (Falkay et al., 1977; Sekiba and Yoshioka, 1979, Ohel et al., 1985), anomalies exist in the reports on NADPH-supported reaction. Thus, Arvela et al. (1976) could not detect significant lipid peroxidation when rat, rabbit and guinea-pig placental microsomes were incubated with NADPH. Similar negative findings were reported by Juchau and Zachariah (1975) for human placental microsomes. In contrast to these negative reports, Diamant et al. (1980) observed accumulation of malondialdehyde (MDA) when human placental microsomes or mitochondria were incubated in the presence of NADPH and iron.

The evidence presented in this paper demonstrates that microsomes of human term placenta can undergo $\mathrm{NADPH}-\mathrm{Fe}^{2+}$-dependent lipid peroxidation. 
Furthermore, our data suggest that the failure of previous researchers (Juchau and Zachariah, 1975, Arvela et al., 1976) to observe lipid peroxidation in placental tissue may be related to improper assay conditions employed. A brief account of this investigation was reported earlier (Kenel and Kulkarni, 1982).

\section{MATERIALS AND METHODS}

\section{Chemicals}

NADPH, Tris- $\mathrm{HCl}$, disodium ethyleneglycol-bis $(\beta$ aminoethylether) $N, N, N^{\prime}, N^{\prime}$-tetraacetic acid (EGTA), ethylenediaminetetraacetic acid (EDTA), 2-thiobarbituric acid (TBA) and sucrose were purchased from Sigma Chemical Co., St Louis, Mo. All other chemicals used were of the highest analytical grade available commercially.

\section{Isolation of microsomes}

Human term placentas from healthy women were placed on ice soon after cesarean delivery. Microsomes were isolated as described previously (Kulkarni et al., 1985) by differential centrifugation of $20 \%$ homogenates prepared in an isolation medium containing: $0.02 \mathrm{M}$ Tris $-\mathrm{HCl}$ buffer, pH 7.4, 0.25 M sucrose, and $0.5 \mathrm{mM}$ either disodium-EGTA or -EDTA. Microsomal suspensions $(2.0 \mathrm{mg} / \mathrm{ml})$, stored up to 2 weeks at $-20^{\circ} \mathrm{C}$ in IM, were diluted at least 4-fold with $0.02 \mathrm{M}$ Tris- $\mathrm{HCl}$ buffer, $\mathrm{pH} 7.4$ and resedimented by centrifugation at $105,000 \mathrm{~g}$ for $60 \mathrm{~min}$ to remove the chelating agent and to minimize the cytosolic contaminants. Washed microsomes were suspended in $0.02 \mathrm{M}$ Tris- $\mathrm{HCl}$ buffer, pH 7.4 and used immediately.

\section{$\mathrm{NADPH}-\mathrm{Fe}^{2+}$ stimulated lipid peroxidation}

Based on preliminary experiments, optimal assay conditions were established. Except as noted otherwise, the standard reaction mixture $(1.0 \mathrm{ml}$ final volume $)$ contained $0.2 \mathrm{mg}$ microsomal protein, $0.4 \mathrm{mM} \mathrm{NADPH}$, and $50 \mu \mathrm{M}$ disodium-EDTA premixed with $75 \mu \mathrm{m} \mathrm{FeCl}_{2}$ in $0.02 \mathrm{M}$ Tris-- $\mathrm{HCl}$ buffer, $\mathrm{pH}$ 7.4. After pre-incubation for $5 \mathrm{~min}$ at $37^{\circ} \mathrm{C}$, the reaction was initiated with the addition of NADPH. Reaction mixtures without NADPH served as controls. After incubation at $37^{\circ} \mathrm{C}$ for the pre-determined time, the reaction was terminated by the rapid addition of $1.0 \mathrm{ml}$ of $35 \%$ trichloroacetic acid containing $4 \mathrm{~N} \mathrm{HCl}$ followed by $50 \mu \mathrm{l}$ of $0.5 \mathrm{mM}$ butylated hydroxytoluene (BHT) in acetone and $2.0 \mathrm{ml}$ of $0.75 \% \mathrm{TBA}$. The amount of TBA-reactive substances (primarily MDA) formed was measured spectrophotometrically as described previously (Kulkarni and Hodgson, 1981).

\section{Other assays}

Microsomal NADPH-cytochrome $c$ reductase activity was assayed essentially as described previously (Robaker $e t$ al., 1981) in the absence of cyanide. Epinephrine oxidation to adrenochrome, an index of $\mathrm{O}_{2}^{-}$generation (Misra and Fridovich, 1972), was measured at $37^{\circ} \mathrm{C}$ spectrophotometrically. The reaction mixtures $(3.0 \mathrm{ml}$ final volume $)$ contained $20 \mathrm{mM}$ Tris buffer, pH 7.4, $0.2 \mathrm{mM}$ epinephrine and $\mathrm{FeCl}_{2}(75 \mu \mathrm{M})$ pre-mixed with EDTA $(50 \mu \mathrm{M})$. The reaction was initiated by the addition of EDTA- $\mathrm{Fe}^{2+}$ to the sample cuvette. Equal volume of Tris buffer was added to the reference cuvette. Magnetic stirrers were used to avoid anaerobiosis. The rates of adrenochrome formation were determined from the linear portion of the reaction curve (see text for further details). Protein content was determined by the biuret method using bovine serum albumin fraction $V$ as a standard (Gornall et al., 1949).

\section{RESULTS AND DISCUSSION}

Without any exception and irrespective of whether EGTA or EDTA was present in the initial tissue homogenization medium, NADPH-supported lipid peroxidation reaction was observed in all the samples of washed microsomes of different placentas, when examined under the standard assay conditions described in the Materials and Methods.

In general, the NADPH-supported basal activity was low in microsomes isolated from placentas homogenized in buffer containing calcium chelator, EGTA (data not shown). This basal activity varied considerably between different microsomal preparations and did not require exogenous iron. The addition of EDTA-Fe ${ }^{2+}$, however, caused further stimulation of lipid peroxidation. It is believed that this basal activity may be due to trace amounts of contaminating iron and/or other reduced transition metal cations present in the reagents used. Iron was required to observe lipid peroxidation since excess EDTA (0.1-1.0 mM) inhibited the basal activity. Similar results were observed for the EDTA$\mathrm{Fe}^{2+}$-dependent reaction (Table 1). Only negligible or no MDA accumulation was observed in the absence of chelated iron when microsomes isolated from the homogenates prepared in buffer containing EDTA were used. In the EDTA-Fe ${ }^{2+}$ - and NADPHsupplemented incubation media, the rates of MDA formation were essentially the same irrespective of whether microsomes isolated from homogenates prepared in buffer containing either EGTA or EDTA were used. No significant decrease in lipid peroxidation rates were observed when microsomes stored up to 2 weeks at $20^{\circ} \mathrm{C}$ were used (data not shown). In the subsequent experiments, lipid peroxidation was assayed using EDTA-Fe ${ }^{2+}$ supplemented reaction media.

Using the standard assay conditions, NADPHsupported $\mathrm{Fe}^{2+}$-dependent lipid peroxidation was found to be linear up to 5 min (Fig. 1) and a plateau in the response curve was evident after about $15 \mathrm{~min}$. In subsequent experiments, only the time points (usually $5 \mathrm{~min}$ ) exhibiting linearity with respect to MDA accumulation were used. Early departure from

Table 1. The effects of different agents on the placental lipid peroxidation*

\begin{tabular}{lcc}
\hline Agent (concentration) & $\begin{array}{c}\text { No. of } \\
\text { observations }\end{array}$ & $\begin{array}{c}\text { Relative specific } \\
\text { activity (\%), } \\
\text { mean } \pm \text { SE }\end{array}$ \\
\hline None & 6 & 100 \\
EDTA $(1.0 \mathrm{mM})$ & 3 & $3 \pm 1$ \\
BHA $(0.1 \mathrm{mM})$ & 3 & $0 \pm 0$ \\
BHT $(0.1 \mathrm{mM})$ & 3 & $4 \pm 2$ \\
HgCl $(0.1 \mathrm{mM})$ & 3 & $7 \pm 3$ \\
Cytochrome $c(0.1 \mathrm{mM})$ & 3 & $15 \pm 3$ \\
Superoxide dismutase $(50 \mu \mathrm{g})$ & 3 & $108 \pm 5$ \\
Catalase $(2000$ Units/ml) & 4 & $83 \pm 12$ \\
Mannitol $(0.1 \mathrm{M})$ & 4 & $92 \pm 11$ \\
Sodium formate $(0.25 \mathrm{M})$ & 4 & $42 \pm 15$ \\
Thiourea $(0.1 \mathrm{M})$ & 4 & $0 \pm 0$ \\
B-carotene $(25 \mu \mathrm{M})$ & 4 & $41 \pm 15$ \\
Vitamin E $(10 \mu \mathrm{M})$ & 4 & $85 \pm 16$ \\
Diphenylisobenzofuran (1 mM) & 4 & $66 \pm 2$ \\
1,4-Diazabicyclo-(2,2,2)octane & 4 & $0 \pm 0$ \\
(50 mM) & & \\
\hline
\end{tabular}

*The range of specific activity observed in the absence of modifiers varied from 2 to $6 \mathrm{nmol} / \mathrm{min} / \mathrm{mg}$ protein in different experiments. Separate incubation medium containing the test chemical but no NADPH served as respective control. Washed microsomes isolated from placental homogenates containing EDTA were used. 


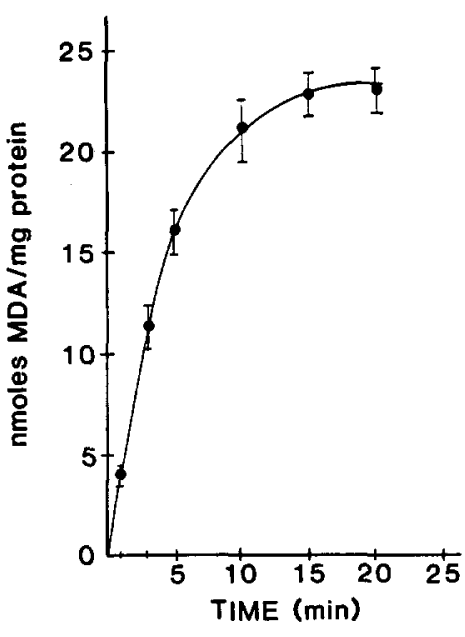

Fig. 1. Time dependence of human placental microsomal lipid peroxidation. The assays were performed in the presence of $0.4 \mathrm{mM} \mathrm{NADPH}$ and EDTA-Fe ${ }^{2+}$ and $0.2 \mathrm{mg}$ of microsomal protein as described in Materials and Methods.

Each point represents the mean $\pm \mathrm{SE}$ of mean $(n=4)$.

linearity suggests that the availability of substrate may be rate limiting, while the lack of an initial lag may be due to the presence of very low concentrations (if any) of endogenous antioxidants in the placental microsomes. No MDA accumulation was observed when boiled microsomes were used, thus suggesting an enzymatic nature of the lipid peroxidation process.

It should be noted that under the assay conditions employed, the observed initial rates of lipid peroxidation varied between 2-6 nmol MDA produced/ $\mathrm{min} / \mathrm{mg}$ protein (about 3-fold inter-individual variation). These values are up to 10 times higher than those reported by Diamant et al. (1980). These investigators used a chelated-iron complex consisting of 4.0 mM ADP and $0.1 \mathrm{mM} \mathrm{FeCl}_{3}$. It is noteworthy that Juchau and Zachariah (1975) also evaluated human placental microsomal lipid peroxidation in the presence of $\mathrm{ADP}-\mathrm{Fe}^{3+}$. In our investigation, the specific activities observed in the presence of 4.0 mM ADP and $0.1 \mathrm{mM} \mathrm{FeCl}_{3}$ were only $10-20 \%$ of those in the presence of EDTA-Fe ${ }^{2+}$. This is probably due to inability of NADPH-cytochrome $P-450$ reductase to effect reduction of $\mathrm{ADP}-\mathrm{Fe}^{3+}$ complex in significant amounts (Morehouse et al., 1984). However, this flavoprotein apparently is capable of EDTA-Fe ${ }^{3+}$ reduction. Falkay et al. (1977), working with human uterine and placental microsomes, reported that ascorbate-supported nonenzymatic lipid peroxidation rate is greater in the presence of $\mathrm{Fe}^{2+}$ than that observed in the presence of $\mathrm{Fe}^{3+}$. In view of these reports, it appears that the higher rates of lipid peroxidation observed in the present study are likely to be due to the use of EDTA-Fe ${ }^{2+}$ complex.

Inspection of the data given in Fig. 2 suggests that the magnitude of MDA accumulation decreases with increasing protein concentration above an optimal concentration of about $0.2 \mathrm{mg} / \mathrm{ml}$. These results concur with the observations of Diamant et al. (1980). In contrast to this, a protein concentration of $4.8 \mathrm{mg} / \mathrm{ml}$

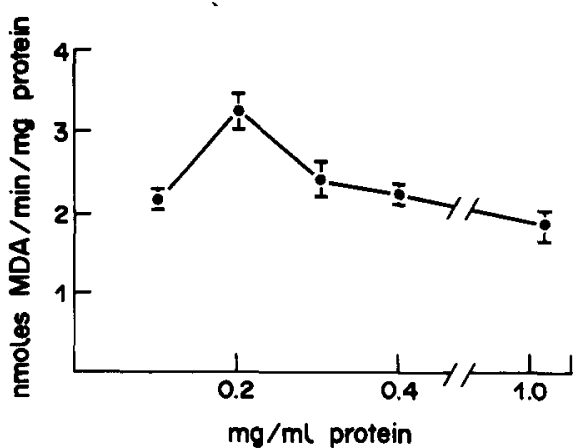

Fig. 2. Influence of protein concentration on lipid peroxidation of human placental microsomes. The assays were performed in the presence of $0.4 \mathrm{mM} \mathrm{NADPH}$ and EDTA$\mathrm{Fe}^{2+}$ and indicated amount of microsomal protein as described in Materials and Methods. Each point represents the mean $\pm \operatorname{SE}(n=4)$

was used in the previous study while evaluating NADPH-dependent lipid peroxidation in the placental microsomes (Juchau and Zachariah, 1975). Although the reasons for the observed decrease in the rates of lipid peroxidation at higher protein concentrations are not clear, it may be due to the presence of endogenous inhibitors in the placental microsomes. This contention is further supported by the recent report of Ohel et al (1985). These investigators observed that when placental microsomes were allowed to undergo the ascorbate dependent non-enzymatic lipid peroxidation, there was a decrease in MDA accumulation with an increase in protein concentration. Chemical identity of the endogenous inhibitor(s), present in human placental microsomes (if any) is unknown at present.

NADPH was required for the lipid peroxidation process and an accumulation of appreciable amounts of MDA was observed in the presence of the lowest concentration (50 $\mu \mathrm{M})$ of NADPH tested (Fig. 3). A pH optimum of 7.4 was observed (Fig. 4) which is not in agreement with the $\mathrm{pH}$ optimum of 6.0 reported by Diamant et al. (1980). This discrepancy may be related to the fact that although the sample absorbance of the NADPH-supplemented incubation

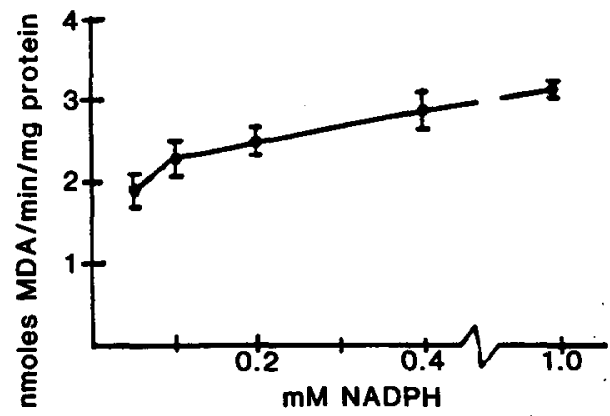

Fig. 3. Effect of NADPH concentration on lipid peroxidation of human placental microsomes. The assays were performed in the presence of EDTA-Fe ${ }^{2+}, 0.2 \mathrm{mg}$ of microsomal protein and the indicated concentration of NADPH as described in Materials and Methods. Each point represents the mean $\pm \operatorname{SE}(n=4)$. 


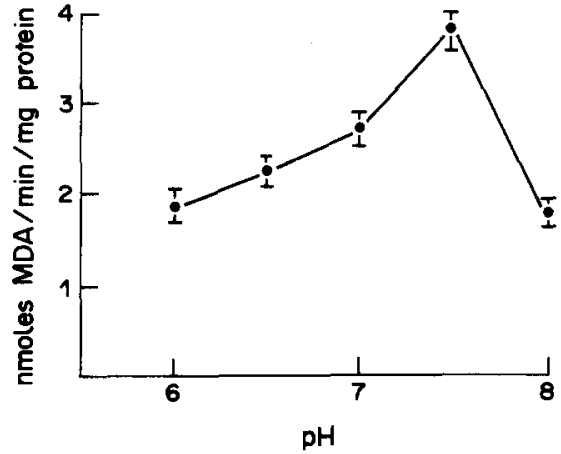

Fig. 4. Effect of $\mathrm{pH}$ on lipid peroxidation of human placental microsomes. The assays were performed in the presence of buffer of desired $\mathrm{pH}, 0.4 \mathrm{mM}$ NADPH, EDTA- $\mathrm{Fe}^{2+}$ and $0.2 \mathrm{mg}$ of microsomal protein as described in Materials and Methods. Each point represents the mean $\pm \operatorname{SE}(n=4)$.

medium was greater at $\mathrm{pH}<7.4$, the absorbance of respective control was also elevated, yielding lower values for the NADPH-Fe ${ }^{2}$-dependent reaction.

The effects of several modulators of lipid peroxidation were tested and the results are given in Table 1. Essentially complete inhibition of lipid peroxidation process was noted in the presence of either $\mathrm{HgCl}_{2}$, an inhibitor of NADPH-cytochrome $P-450$ reductase (Kulkarni and Hodson, 1980) or cytochrome $c$, an exogenous electron acceptor which competes for the reducing equivalents. This suggests that a continuous electron input via NADPHcytochrome $P-450$ reductase to reduce $\mathrm{Fe}^{3+}$ to $\mathrm{Fe}^{2+}$ is necessary in order to observe placental lipid peroxidation process. In accord with many reports (Aust et al., 1985), the inhibition observed with antioxidants BHA and BHT suggests that the placental lipid peroxidation is a radical generating process.

The ability of placental microsomes, isolated from the EDTA containing homogenization medium, to generate $\mathrm{O}_{2}^{--}$was assessed indirectly by monitoring epinephrine oxidation. The oxidation of epinephrine by placental microsomes was not detectable in the presence of NADPH alone. A small but measurable epinephrine oxidation was observed when EDTA$\mathrm{Fe}^{2+}$ was incorporated into the reaction medium. These results are in agreement with those reported by Morehouse et al. (1984). For the following reasons, these results do not necessarily mean that under the experimental conditions employed, placental microsomes (most likely NADPH-cytochrome $P-450$ reductase and/or cytochrome $P-450$ ) do not generate $\mathrm{O}_{2}^{-}:$(1) alkaline conditions $(\mathrm{pH} \geqslant 8.5)$ are necessary to detect significant rates of $\mathrm{O}_{2}^{-}$generation by the NADPH-cytochrome $P-450$ reductase (Aust et al., 1972) whereas our assays were performed at $\mathrm{pH} 7.4$; (2) not all the $\mathrm{O}_{2}^{--}$generated in the membrane diffuses out into the aqueous environment; (3) all the molecules of $\mathrm{O}_{2}^{--}$escaping from the membrane do not necessarily participate in epinephrine oxidation and (4) in aqueous medium, dismutation of $\mathrm{O}_{2}^{--}$to $\mathrm{H}_{2} \mathrm{O}_{2}$ and oxygen may escape its detection.

Despite many attempts, the role of reduced oxygen species in microsomal lipid peroxidation is not completely understood. To investigate the role of oxygen reduction, we evaluated the effects of various scavengers of reactive oxygen species on the microsomal lipid peroxidation. The results of these experiments are presented in Table 1. Both superoxide dismutase and catalase were found to be ineffective in blocking the lipid peroxidation process. It is highly unlikely that these results are due either to inadequate amounts of the superoxide dismutase and catalase used or inaccessibility of $\mathrm{O}_{2}^{-}$and $\mathrm{H}_{2} \mathrm{O}_{2}$ in the aqueous environment to superoxide dismutase and catalase. The results, in fact, favor the proposition by others (Aust et al., 1985) that both $\mathrm{O}_{2}^{-}$and $\mathrm{H}_{2} \mathrm{O}_{2}$ may not possess the necessary reactivity to initiate lipid peroxidation directly.

While neither $\mathrm{O}_{2-}^{-}$nor $\mathrm{H}_{2} \mathrm{O}_{2}$ seems to be the initiator of lipid peroxidation, both of these reactive oxygen species may be obligatory intermediates, i.e. precursors of the ultimate initiating species. The fact that lipid peroxidation can only be observed in the presence of iron or other reduced transitional metals implies that ${ }^{\circ} \mathrm{OH}$ and/or ${ }^{1} \mathrm{O}_{2}$, generated via HaberWeiss reaction or other mechanisms, may be responsible. However, the results (Table 1) obtained in the presence of scavengers of ${ }^{\circ} \mathrm{OH}$ were inconclusive. Thus, mannitol and benzoate were ineffective while formate caused some inhibition of lipid peroxidation. On the other hand, thiourea, at the concentrations used, completely blocked the reaction. Since Tris buffer was used in these assays and it can interact with the ${ }^{\circ} \mathrm{OH}$ generated in the incubation medium (Halliwell and Ahluwalia, 1976), a precise evaluation of the role of this highly reactive oxygen species is difficult. Similar variability in results was exhibited by the scavengers of ${ }^{1} \mathrm{O}_{2}$ (Table 1). These observations may, in part, be due to the lack of specificity of the scavengers (Kulkarni and Hodgson, 1981). Alternately, it means that the generation of these reactive oxygen species occurs in the hydrophobic domain of the membrane which is inaccessible to the scavengers used. The other possibility is that lipid peroxidation may be triggered by some other species such as ferrous-dioxygen-ferric complex of yet undefined structure and reactivity proposed previously by some investigators (Aust et al., 1985).

Since a decrease in the rat liver mitochondrial lipid peroxidation by phosphate ions was reported earlier (Hunter et al., 1963), the possibility of similar inhibition of human placental microsomal lipid peroxidation was suspected. The results presented in the Fig. 5 clearly indicate an extreme sensitivity of the placental lipid peroxidation process to the phosphate buffer. Essentially similar observations have recently been reported for lipid peroxidation of rat hepatic microsomes (Tan et al., 1984). From these results it appears that the failure of Juchau and Zachariah (1975) and Arvela et al. (1976) to observe NADPHsupported lipid peroxidation in placental microsomes might have been due to their use of phosphate buffer in the incubation media.

Experiments were performed to elucidate the possible mechanism involved in the inhibition of lipid peroxidation by phosphate ions. In Tris buffer, the specific activity of $98.06 \pm 26.32 \mathrm{nmol}$ cytochrome $c$ reduced $/ \mathrm{min} / \mathrm{mg}$ microsomal protein $(n=6)$ was observed for NADPH-cytochrome $c$ reductase. These results on the basal NADPH-cytochrome $c$ reductase 


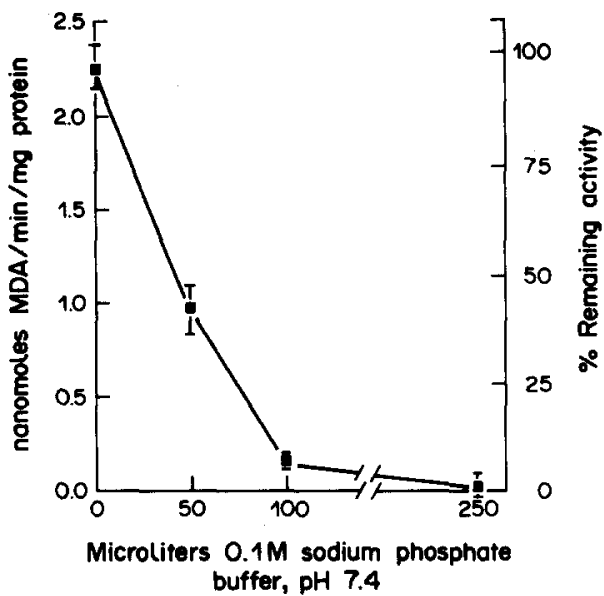

Fig. 5. Inhibition of human placental microsomal lipid peroxidation by phosphate. The assays were performed using incubation media in which Tris buffer was substituted with the indicated amounts of $0.1 \mathrm{M}$ sodium phosphate buffer, pH 7.4, 0.4 mM NADPH and EDTA-Fe ${ }^{2+}$ and $0.2 \mathrm{mg}$ of microsomal protein. Each point represents the mean $\pm \operatorname{SE}(n=4)$. See text for further details.

activity are comparable to those reported in the literature for the human placental microsomes (Juchau and Zachariah, 1975). The addition of increasing amounts $(50-250 \mu \mathrm{l} / \mathrm{ml})$ of $0.1 \mathrm{M} \mathrm{Na}$ phosphate buffer, pH 7.4 to the incubation medium did not cause a statistically significant change in this activity. This suggests that phosphate ions do not hinder the electron flow and that reductase is not the locus of inhibition of lipid peroxidation by phosphate ions.

In view of these results, a possible interference with the redox cycling of iron was investigated indirectly by monitoring the superoxide generation in a model system. To avoid complications in the interpretation of data, the experiments were performed in the absence of placental microsomes. The preparation of chelated iron in phosphate buffer was not successful because it resulted in the formation of precipitate (possibly insoluble ferrous phosphate). Similar observations were also reported by Flitter et al. (1983). The epinephrine oxidation curves during the first $60-90 \mathrm{sec}$ were non-linear and indicated that the initial rates of adrenochrome formation are, in general, lower in the phosphate containing reaction media. This was probably due to the gradual reaction of phosphate ions with iron. Subsequently, the reaction was linear for several minutes. The results (Table 2) indicated a marked decrease in the steady state superoxide formation in the presence of increasing amounts of phosphate buffer. It appears, that possibly, it is not the redox cycling per se but the decreased amounts of the chelated iron available for redox cycling may be responsible for the observed inhibition of lipid peroxidation by phosphate ions.

Studies on further characterization of the placental lipid peroxidation process and its involvement in xenobiotic metabolism are in progress in our laboratory.

\section{SUMMARY}

The evidence presented suggests that NADPH-
Table 2. The effect of phosphate buffer on epinephrine oxidation*

\begin{tabular}{cc}
\hline $\begin{array}{c}\text { Phosphate buffer } \\
(\mu \mathrm{l} / \mathrm{ml})\end{array}$ & $\begin{array}{c}\text { Adrenochrome formation } \\
\text { relative activity }\end{array}$ \\
\hline 0 & 100 \\
50 & 45 \\
100 & 22 \\
250 & 11 \\
\hline
\end{tabular}

* Assays were carried out in the absence of placental microsomes as described in Materials and Methods. Each reaction mixture ( $3.0 \mathrm{ml}$ final volume) contained $0.02 \mathrm{M}$ Tris- $\mathrm{HCl}$ buffer, $\mathrm{pH} 7.4$, EDTA-Fe ${ }^{2+}$ (absent in the reference cuvette), $0.2 \mathrm{mM}$ epinephrine and indicated volume of $0.1 \mathrm{M} \mathrm{Na-phosphate} \mathrm{buffer.}$ The reaction was initiated with the addition of EDTA-Fe ${ }^{2+}$. The values given are means of 3 separate experiments. The rates of epinephrine oxidation were estimated from linear portion of the reaction curves.

$\mathrm{Fe}^{2+}$-dependent lipid peroxidation occurs in human placental microsomes. $\mathrm{Fe}^{2+}$ was found to be obligatory and in order to attain maximal rates of lipid peroxidation, the use of relatively low protein concentration, and shorter incubation times are necessary. Human placental microsomal lipid peroxidation was found to be highly sensitive to phosphate buffer. The effectiveness of various scavengers of reactive oxygen species to block lipid peroxidation was evaluated. However, the data were inconclusive and the identity of the initiator of lipid peroxidation remains unknown. It appears that improper assay conditions might have been responsible for the failure of previous researchers (Juchau and Zachariah, 1975; Arvela et al., 1976) to detect this reaction in the placental microsomes.

Acknowledgements - This work was supported, in part, by a Rackham Faculty Research Grant, and by Reproductive Hazards in the Workplace Research Grant No. 15-82 from March of Dime Birth Defects foundation.

\section{REFERENCES}

Arvela P., Karki N. T. and Pelkonen O. (1976) Lipoperoxidation rates and drug-oxidizing enzyme activities in the liver and placenta of some mammal species during the perinatal period. Experimentia 32, 1311-1313.

Aust S. D., Morehouse L. A. and Thomas C. E. (1985) Role of metals in oxygen radical reactions. J. Free Rad. Biol. Med. 1, 3-25.

Aust S. D., Roerig D. L. and Pederson T. C. (1972) Evidence for superoxide generation by NADPHcytochrome $C$ reductase of rat liver microsomes. Biochem. biophys. Res. Commun. 47, 1133-1137.

Benedetti A., Comporti M., Fulceri R. and Esterbauer H. (1984) Cytotoxic aldehydes originating from the lipid peroxidation of liver microsomal lipids. Identification of 4,5-dihydroxydecenal. Biochim. biophys. Acta 792, 172-181.

Bus J. S. and Gibson J. E. (1979) Lipid peroxidation and its role in toxicology. Rev. Biochem. Toxic. 1, 125-149.

Diamant S., Kissilevitz R. and Diamant Y. (1980) Lipid peroxidation system in human placental tissue: General properties and the influence of gestational age. Biol. Reprod. 23, 776-781.

Dix T. A. and Marnett L. J. (1983) Metabolism of polycyclic aromatic hydrocarbon derivatives to ultimate carcinogens during lipid peroxidation. Science 221, 77-79.

Falkay G., Herczeg J. and Sas M. (1977) Microsomal lipid peroxidation in human pregnant uterus and placenta. Biochem. biophys. Res. Commun. 79, 843-851. 
Flitter W., Rowley D. A. and Halliwell B. (1983) Superoxide-dependent formation of hydroxyl radicals in the presence of iron salts. What is the physiological iron chelator? FEBS Lett. 158, 310-312.

Fridovich 1. (1986) Biological effects of the superoxide radical. Archs Biochem. Biophys. 247, 1-11.

Gornall A. G., Bardawill C. J. and David M. M. (1949) Determination of serum proteins by means of the Biuret reaction. J. biol. Chem. 177, 751-760.

Halliwell B. and Ahluwalia S. (1976) Hydroxylation of $p$-coumaric acid by horseradish peroxidase. The role of superoxide and hydroxyl radicals. Biochem. J. 153, $513-518$.

Halliwell B. and Gutteridge J. M. C. (1984) Oxygen toxicity, oxygen radicals, transition metals and disease. Biochem. J. 219, 1-14.

Hunter F. E., Gebicki J. M., Hoffstein P. E., Weinstein J and Scott A. (1963) Swelling and lysis of rat liver mitochondria induced by ferrous iron. J. biol. Chem. 238 , 828-835.

Juchau M. R. (1980) Drug biotransformation in the placenta. Pharmac. Therap. 8, 501-524.

Juchau M. R. and Zachariah P. K. (1975) Comparative studies on the oxidation and reduction of drug substances in human placenta versus rat hepatic microsomes. Biochem. Pharmac. 24, 227-233.

Kenel M. F. and Kulkarni A. P. (1982) Effects of paraquat on the NADPH-dependent lipid peroxidation in human placental microsomes. Joint ASPET/SOT Meeting Louiseville, Kentucky, Abstract No. 429.

Kulkarni A. P. and Hodgson E. (1980) Phenazine methosulfate as a "Bypass Reagent" in the reduction of microsomal cytochromes. In Microsomes, Drug Oxidations and Chemical Carcinogenesis (Edited by Coon M. J., Conney A. H., Estabrook R. W., Gelboin H. V., Gillett J. R. and O'Brien P. J.), pp. 355-358. Academic Press, New York

Kulkarni A. P. and Hodgson E. (1981) A comparison of NADPH and cumene hydroperoxide-stimulated lipid peroxidation in mouse hepatic microsomes. Int. J. Biochem. 13, 811-816

Kulkarni A. P., Strohm B. and Houser W. H. (1985) Human placental indanol dehydrogenase: some properties of the microsomal enzyme. Xenobiotica 15, 513-519.
Misra H. P. and Fridovich I. (1972) The role of superoxide anion in the autooxidation of epinephrine and a simple assay for superoxide dismutase. J. biol. Chem. 247, $3170-3175$.

Morehouse L. A., Thomas C. E. and Aust S. D. (1984) Superoxide generation by NADPH-cytochrome $P-450$ reductase: the effect of iron chelators and the role of superoxide in microsomal lipid peroxidation. Archs Biochem. Biophys. 232, 366-377.

Morgenstern R., DePierre J. W., Lind C., Guthenberg C., Mannervik B. and Ernster L. (1981) Benzo(a)pyrene quinones can be generated by lipid peroxidation and are conjugated with glutathione S-transferase B from rat liver. Biochem. biophys. Res. Commun. 99, 682-690.

Nelson J. L. and Kulkarni A. P. (1986a) Partial purification of human placental peroxidase by affinity chromatography. Toxicologist $6,144$.

Nelson J. L. and Kulkarni A. P. (1986b) Peroxidase activity in the mouse uterus, placenta and fetus during pregnancy. Biochem. Int. 13, 131-138

Ohel G., Kisselevitz R., Margalioth E. J. and Schenker J. G. (1985) Ascorbate-dependent lipid peroxidation in the human placenta and fetal membranes. Gynec. Obstet. Invest. 19, 73-77.

Osimitz T. G. and Kulkarni A. P. (1982) Oxidative metabolism of xenobiotics during pregnancy: Significance of microsomal flavin containing monooxygenase. Biochem. biophys. Res. Commun. 109, 1164-1171.

Plaa G. L. and Witschi H. (1976) Chemicals, drugs and lipid peroxidation. A. Rev. Pharmac. 16, 125-141.

Robaker K. M., Kulkarni A. P. and Hodgson E. (1981) Pesticide induced changes in the mouse hepatic microsomal $P$-450-dependent monooxygenase system and other enzymes. J. envir. Sci. Hlth B 16, 529-545.

Sekiba K. and Yoshioka T. (1979) Changes of lipid peroxidation and superoxide dismutase activity in the human placenta. Am. J. Obstet. Gynec. 135, 368-371.

Slater T. F. (1984) Free-radical mechanisms in tissue injury. Biochem. J. 222, 1-15.

Tan K. H., Meyer D. J., Belin J. and Ketterer B. (1984) Inhibition of microsomal lipid peroxidation by glutathione and glutathione transferases $B$ and AA. Role of endogenous phospholipase $A_{2}$. Biochem. J. 220, 243-252. 